\title{
Prognostic value of copper transporter 1 expression in patients with clear cell renal cell carcinoma
}

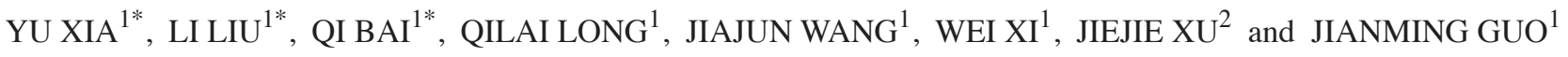 \\ ${ }^{1}$ Department of Urology, Zhongshan Hospital; ${ }^{2}$ Department of Biochemistry and Molecular Biology, \\ School of Basic Medical Sciences, Fudan University, Shanghai 200032, P.R. China
}

Received April 18, 2016; Accepted July 5, 2017

DOI: $10.3892 / \mathrm{ol} .2017 .6942$

\begin{abstract}
Clearcell renal cell carcinoma(ccRCC) features a Von Hippel-Lindau mutation, associated with a hypoxia-inducible factor (HIF) imbalance. Copper transporter 1 (CTR1) may also promote tumor progression through the modulation of the HIF pathway by copper. Therefore, the present study explored the prognostic effect of tumor CTR 1 expression in patients with ccRCC. A total of 293 patients with ccRCC that underwent nephrectomy were retrospectively enrolled. CTR 1 expression was assessed by immunohistochemistry, and its association with clinicopathological features and prognosis were evaluated. The present data indicated that high tumor CTR1 expression was independently associated with poor overall survival (OS) [hazard ratio, 2.291; 95\% confidence interval (CI), 1.389-3.777; $\mathrm{P}<0.001]$ and disease-free survival (DFS) (hazard ratio, 2.210; 95\% CI, 1.299-3.759; $\mathrm{P}=0.003$ ) rates in patients with ccRCC. Furthermore, CTR1 expression was significantly higher for Mayo Clinic stage, size, grade and necrosis score risk groups, and could be incorporated into several existing prognostic models to improve performance. Nomograms incorporating tumor CTR1 expression with other parameters performed well in the 5- and 8-year OS and DFS rate predictions of patients (concordance index 0.805 and 0.787 , respectively). In conclusion, the present study demonstrated that CTR1 expression is a potential independent biomarker for poor prognosis for the recurrence and survival prediction of patients with ccRCC following nephrectomy.
\end{abstract}

Correspondence to: Dr Jiejie Xu, Department of Biochemistry and Molecular Biology, School of Basic Medical Sciences, Fudan University, 138 Yi Xue Yuan Road, Shanghai 200032, P.R. China E-mail: jjxufdu@fudan.edu.cn

Dr Jianming Guo, Department of Urology, Zhongshan Hospital, Fudan University, 180 Feng Lin Road, Shanghai 200032, P.R. China E-mail: guo.jianming@zs-hospital.sh.cn

\section{"Contributed equally}

Key words: clear cell renal cell carcinoma, copper transporter 1, overall survival, disease-free survival, prognostic factor, nomogram

\section{Introduction}

Renal cell carcinoma (RCC) accounts for 2-3\% of all cases of malignancy in adults globally in 2014 (1). Although associated diagnostic techniques and targeted therapies have greatly improved in recent decades, in 2004, 20-30\% of RCC patients were diagnosed with metastatic disease, and a further one-third of patients with localized disease that undergo curative surgery subsequently experience recurrence or metastasis in North America (2). The tumor-node-metastasis (TNM) stage (3) and Fuhrman grade (4) systems remain the most used outcome predictors; several integrated models have also been established, including the Mayo Clinic stage, size, grade and necrosis (SSIGN) score (5) and the University of California Integrated Staging System (UISS) (6); however, these parameters are not entirely reliable (7). In the era of precision medicine, it is expected that specific molecular biomarkers will provide supplementary prognostic information that may be incorporated into existing conventional models for the improved prediction of patient prognosis (8).

Copper transporter (CTR) 1, encoded by the SLC31AI gene, has been characterized as a high-affinity copper transporter since its identification in 1997 (9). This membrane-bound molecule is the major driving force in facilitating copper import, and thus may raise the $\mathrm{Cu}^{2+}$ concentration in expressing cells (10). Copper, as an essential trace mineral, serves an important role in the regulation of human physiological functions; its aberrant upregulation may promote tumor angiogenesis and progression in various types of cancer (11-13). This oncogenic potential is partially associated with the stimulation of the hypoxia-inducible factor (HIF) pathway by copper. For example, a previous study has identified that copper serves an essential role in the HIF-1 $\alpha$ /hypoxia response element-binding process; high concentrations of $\mathrm{Cu}^{2+}$ may stabilize HIF-1 $\alpha$ in the nucleus and activate downstream signals, including the upregulation of vascular endothelial growth factor (VEGF), and induce the epithelial-mesenchymal transition (EMT) (14). CTR1-knockdown, leading to reduced intracellular copper, has been demonstrated to inhibit angiogenesis and EMT in multiple types of cell, including human breast cancer and endothelial cells $(15,16)$. Clinical trials with copper chelation therapy for metastatic diseases have also generated promising data (17). 
In RCC, the most common histological type is clear cell renal cell carcinoma (ccRCC; 70-85\%) (18), which exhibits a Von Hippel-Lindau (VHL) mutation, leading to an activation of HIF signaling (19). Since CTR1 may be associated with cellular copper regulation and interact with the HIF pathway, this molecule may be associated with the outcome of patients with ccRCC. In the present study, the association between CTR1 expression, as determined with IHC, and the survival time of 293 patients with ccRCC was evaluated. Prognostic improvements using CTR1 data with several well-established predictive models were also analyzed, and two nomograms integrating the expression of this molecule with other clinical parameters were formed to predict the overall survival (OS) and disease-free survival (DFS) outcomes for patients.

\section{Materials and methods}

Patients and clinical database. A total of 293 patients (age range, 15-86 years; median age, 55 years; 90 female, 203 male) with ccRCC who underwent nephrectomy were retrospectively recruited from the Department of Urology,Zhongshan Hospital, Fudan University (Fudan, China) between January 2005 and June 2007. All methods in the present study were approved by the Ethics Committee of Zhongshan Hospital (approval no. B2015-030) and were performed in accordance with the committee guidelines. Written informed consent was obtained from all individual patients included in the present study. The inclusion criteria were as follows: No history of other malignant tumors, no history of targeted therapy prior to or following surgery, and pathologically determined ccRCC. Patients with mixed-type renal cancer, bilateral renal cancer, tumor necrosis area $>80 \%$ or those with perioperative morbidity were excluded.

The median follow-up for all available patients was 99.10 months (range, 2.63-120.47 months) and the follow-up interval was 3 months, until January 30, 2015. Metastasis or recurrences were defined based on imaging tests or histopathology information. Age, sex, tumor size, TNM stage, Fuhrman grade, tumor necrosis and Eastern Cooperative Oncology Group performance status (ECOG PS) (20) information for patients was obtained and is included in Table I. Tumor histological type and differentiation were reassessed by two urological pathologists according to the 2004 World Health Organization criteria (21). Tumor stage was reclassified according to chest radiography, abdominal computerized tomography and pathological reports of patients based on the 2010 American Joint Committee on Cancer TNM classification (3). Tumor size was measured as the longest diameter in the pathological information. Tumor necrosis was defined as the presence of microscopic coagulative necrosis.

OS time was calculated from the date of nephrectomy to the date of mortality the last follow-up. DFS time was defined as the time from nephrectomy to disease recurrence or the last follow-up time; mortalities occurring without disease recurrence were considered to be censored. The SSIGN score was applied to classify patients into three risk levels: 0-3 (low), 4-7 (intermediate) and $\geq 8$ (high) in OS analyses (5,22). Similarly, patients were classified into three risk levels for DFS analyses: 0-2 (low), 3-5 (intermediate) and $\geq 6$ (high), based on SSIGN (localized) score, as previously reported (23).
IHC and evaluation. Immunohistochemical staining was performed on a tissue microarray, as previously described (24). For each primary tumor block, two representative cores from areas without necrosis and hemorrhage were selected for analysis. A rabbit polyclonal antibody against human CTR1 (cat. no. ab133385; dilution, 1:100; Abcam, Cambridge, MA, USA) and the EnVision Detection System (Dako; Agilent Technologies, Inc., Santa Clara, CA, USA) were applied in the procedure. The specificity of the anti-CTR1 antibody was confirmed by western blot procedure, performed as previously described (25) (anti-CTR1 antibody dilution, 1:1,000). The RCC cell lines ACHN and 786-O were purchased from the Type Culture Collection of the Chinese Academy of Sciences (Shanghai, China). As a negative control, similar IHC procedures were applied to tissue samples without applying the primary antibody.

An Olympus camera (Olympus Corporation, Tokyo, Japan), a Nikon eclipse Ti-s microscope (magnification, x200; Nikon Corporation, Tokyo Japan) and NIS-Elements F3.2 software (Nikon Corporation) were used in recording the staining results. The three images with the strongest staining were obtained from each core and the associated integrated optical density (IOD) scores were calculated with Image-Pro Plus version 6.0 software (Media Cybernetics Inc., Rockville, MD, USA). The combined IOD mean of the six images from two cores was regarded as the final staining intensity for each block used for further statistical analysis. These slides were evaluated by two experienced pathologists that were unaware of the clinical features and outcomes of patients.

Statistical analysis. For determining CTR1 high/low expression, the IOD score cut-point was evaluated by X-tile software (version 3.6.1) (26) through the minimum P-value method. $\chi^{2}$ test, Fisher's exact method and Cochran-Mantel-Haenszel $\chi^{2}$ test were applied for assessing the association between CTR1 expression and the clinicopathological parameters of patients. Survival curves for OS and DFS were estimated using the Kaplan-Meier method and analyzed by log-rank test. Cox univariate analysis was performed and parameters with statistical significance were brought into a multivariate Cox proportional hazards model. Harrell's concordance index (c-index) and Akaike's information criterion (AIC) were generated to compare the predictive ability of various models with or without the addition of CTR1 (27). GraphPad Prism 6 (GraphPad Software, Inc., La Jolla, CA, USA), SPSS 21.0 (IBM SPSS, Armonk, NY, USA) and Stata (version 12.1; StataCorp LP, College Station, TX, USA) were used in these procedures. $\mathrm{P}<0.05$ was considered to indicate a statistically significant difference. For nomogram formation and calibrations, R software version 3.1.2 with the 'rms' package ( $\mathrm{R}$ Foundation for Statistical Computing, Vienna, Austria) was applied and the c-index was used for measuring its prognostic accuracy.

\section{Results}

Immunohistochemical CTR1 intensity and its association with clinicopathological characteristics. CTR1 expression was predominantly observed in the cytoplasm and/or membrane in IHC. The staining intensity was divided into low expression and high expression based on the cutoff value $(9,500)$ derived 
Table I. Clinical characteristics of patients according to CTR1 expression.

\begin{tabular}{|c|c|c|c|c|}
\hline \multirow[b]{2}{*}{ Characteristics } & \multirow[b]{2}{*}{ Patients, $\mathrm{n}$} & \multicolumn{2}{|c|}{ CTR1 expression, $\mathrm{n}$} & \multirow[b]{2}{*}{ P-value } \\
\hline & & Low & High & \\
\hline All patients & 293 & 177 & 116 & \\
\hline Age, years & & & & $0.077^{\mathrm{a}}$ \\
\hline$\leq 55$ & 145 & 95 & 50 & \\
\hline$>55$ & 148 & 82 & 66 & \\
\hline Sex & & & & $0.870^{\mathrm{a}}$ \\
\hline Female & 90 & 55 & 35 & \\
\hline Male & 203 & 122 & 81 & \\
\hline Tumor size, $\mathrm{cm}$ & & & & $0.576^{\mathrm{a}}$ \\
\hline$\leq 4$ & 165 & 102 & 63 & \\
\hline$>4$ & 128 & 75 & 53 & \\
\hline $\mathrm{T}$ classification & & & & $<0.001^{\mathrm{b}}$ \\
\hline $\mathrm{T} 1$ & 185 & 132 & 53 & \\
\hline $\mathrm{T} 2$ & 27 & 13 & 14 & \\
\hline T3 & 77 & 31 & 46 & \\
\hline $\mathrm{T} 4$ & 4 & 1 & 3 & \\
\hline $\mathrm{N}$ classification & & & & $0.489^{\mathrm{a}}$ \\
\hline No & 35 & 17 & 18 & \\
\hline N1 & 2 & 0 & 2 & \\
\hline $\mathrm{Nx}$ & 256 & & & \\
\hline Distant metastasis & & & & $0.726^{\mathrm{a}}$ \\
\hline No & 277 & 168 & 109 & \\
\hline Yes & 16 & 9 & 7 & \\
\hline Tumor, node, metastasis stage & & & & $<0.001^{\mathrm{b}}$ \\
\hline I & 179 & 128 & 51 & \\
\hline II & 23 & 10 & 13 & \\
\hline III & 71 & 29 & 42 & \\
\hline IV & 20 & 10 & 10 & \\
\hline Fuhrman grade & & & & $0.003^{\mathrm{b}}$ \\
\hline 1 & 31 & 20 & 11 & \\
\hline 2 & 217 & 140 & 77 & \\
\hline 3 & 42 & 17 & 25 & \\
\hline 4 & 3 & 0 & 3 & \\
\hline Necrosis & & & & $0.194^{\mathrm{a}}$ \\
\hline Absent & 252 & 156 & 96 & \\
\hline Present & 41 & 21 & 20 & \\
\hline Eastern Cooperative Oncology Group performance status & & & & $0.018^{\mathrm{b}}$ \\
\hline 0 & 214 & 137 & 77 & \\
\hline 1 & 64 & 33 & 31 & \\
\hline 2 & 11 & 7 & 4 & \\
\hline 3 & 4 & 0 & 4 & \\
\hline
\end{tabular}

${ }^{\mathrm{a}} \chi^{2}$ test or Fisher's exact test. ${ }^{\mathrm{b}}$ Cochran-Mantel-Haenszel $\chi^{2}$ test. $\mathrm{P}<0.05$ was considered to indicate a statistically significant difference. CTR1, copper transporter 1 .

from total IOD score (median, 9,040; interquartile range, 7,291-11,038; mean \pm standard deviation, 9,267 $\pm 3,129)$ using the 'minimum P-value' approach (Fig. 1A and B). Patients were subsequently separated into CTR1 low $(n=177)$ and
CTR1 high ( $n=116)$ groups (Fig. 1C). The detailed characteristics of patients and the association between CTR 1 expression and clinicopathological features are included in Table I. CTR1 expression was significantly associated with $\mathrm{T}$ classification 
Table II. Univariate analyses of characteristics associated with OS and DFS.

\begin{tabular}{|c|c|c|c|c|c|c|}
\hline \multirow[b]{2}{*}{ Variables } & \multicolumn{3}{|c|}{ OS $(n=293)$} & \multicolumn{3}{|c|}{ DFS (n=268) } \\
\hline & HR & $95 \% \mathrm{CI}$ & P-value ${ }^{a}$ & HR & $95 \% \mathrm{CI}$ & P-value ${ }^{a}$ \\
\hline \multicolumn{7}{|l|}{ Age, years } \\
\hline$>55$ vs. $\leq 55$ & 2.076 & $1.322-3.260$ & 0.002 & 1.740 & $1.084-2.794$ & 0.022 \\
\hline \multicolumn{7}{|l|}{ Sex } \\
\hline Male vs. female & 1.044 & $0.653-1.669$ & 0.857 & 0.950 & $0.578-1.562$ & 0.841 \\
\hline \multicolumn{7}{|l|}{ Tumor size, $\mathrm{cm}$} \\
\hline$>4$ vs. $\leq 4$ & 2.045 & $1.320-3.168$ & 0.001 & 2.339 & $1.456-3.755$ & $<0.001$ \\
\hline T classification & & & $<0.001$ & & & $<0.001$ \\
\hline $\mathrm{T} 1$ & RV & - & - & $\mathrm{RV}$ & - & - \\
\hline $\mathrm{T} 2$ & 3.340 & $2.066-5.402$ & $<0.001$ & 3.626 & $1.769-7.432$ & $<0.001$ \\
\hline $\mathrm{T} 3$ & 3.542 & $1.857-6.756$ & $<0.001$ & 3.004 & $1.784-5.057$ & $<0.001$ \\
\hline $\mathrm{T} 4$ & 7.946 & $2.420-26.092$ & 0.001 & 15.514 & $5.350-44.983$ & $<0.001$ \\
\hline \multicolumn{7}{|l|}{$N$ classification $(n=44)$} \\
\hline N1 vs. N0 & 1.145 & $0.150-8.728$ & 0.896 & - & - & - \\
\hline \multicolumn{7}{|l|}{ Distant metastasis } \\
\hline Yes vs. no & 5.390 & $2.918-9.956$ & $<0.001$ & - & - & - \\
\hline Tumor, node, metastasis stage & & & $<0.001$ & & & $<0.001$ \\
\hline I & RV & - & - & RV & - & - \\
\hline II & 3.279 & $1.592-6.756$ & 0.001 & 3.875 & $1.938-7.751$ & $<0.001$ \\
\hline III & 3.403 & $2.031-5.702$ & $<0.001$ & 2.920 & $1.726-4.940$ & $<0.001$ \\
\hline IV & 9.429 & $5.023-17.698$ & $<0.001$ & 15.532 & $5.356-45.039$ & $<0.001$ \\
\hline Fuhrman grade & & & $<0.001$ & & & $<0.001$ \\
\hline $1-2$ & RV & - & - & RV & - & - \\
\hline 3 & 3.187 & $1.971-5.152$ & $<0.001$ & 3.478 & $2.061-5.868$ & $<0.001$ \\
\hline 4 & 4.367 & $1.366-13.962$ & 0.013 & 4.968 & $1.545-15.977$ & 0.007 \\
\hline \multicolumn{7}{|l|}{ Necrosis } \\
\hline Present vs. absent & 2.699 & $1.656-4.401$ & $<0.001$ & 3.081 & $1.834-5.175$ & $<0.001$ \\
\hline Eastern Cooperative Oncology Group performance status & & & $<0.001$ & & & $<0.001$ \\
\hline 0 & RV & - & - & RV & - & - \\
\hline 1 & 3.167 & $2.005-5.003$ & $<0.001$ & 2.706 & $1.629-4.494$ & $<0.001$ \\
\hline 2 & 2.148 & $0.770-5.990$ & 0.144 & 2.093 & $0.467-6.768$ & 0.217 \\
\hline 3 & 8.667 & $3.078-24.405$ & $<0.001$ & 9.471 & $3.350-26.778$ & $<0.001$ \\
\hline \multicolumn{7}{|l|}{ Tumor CTR 1 expression } \\
\hline High vs. low & 3.636 & $2.305-5.736$ & $<0.001$ & 3.933 & $2.403-6.436$ & $<0.001$ \\
\hline
\end{tabular}

${ }^{a}$ Data obtained from the Cox proportional hazards model. P-value $<0.05$ was regarded as statistically significant. OS, overall survival; DFS, disease-free survival; HR, hazard ratio; CI, confidence interval; RV, reference value; CTR1, copper transporter 1.

$(\mathrm{P}<0.001)$, TNM stage $(\mathrm{P}<0.001)$, Fuhrman grade $(\mathrm{P}=0.003)$ and ECOG PS $(\mathrm{P}=0.018)$.

Kaplan-Meier and subgroup analysis to assess the prognostic value of CTR1 in patients with ccRCC. Within the cohort, $28.3 \%(83 / 293)$ patients succumbed to any cause during the follow-up period. Subsequent to excluding 25 patients with pre-operational metastasis or without recurrence information, $26.5 \%(71 / 268)$ patients developed disease recurrence in the present study. Kaplan-Meier curves revealed that patients with ccRCC with high CTR1 expression had a significantly poorer OS ( $\mathrm{P}<0.001$; Fig. 2A) and DFS ( $\mathrm{P}<0.001$; Fig. 2B) rate compared with those with low CTR1 expression. To investigate whether this was dependent on the SSIGN score, a subgroup analysis was performed on the overall cohort. Fig. 2C and D demonstrate that the proportion of high CTR1 expression specimens was elevated with an increasing SSIGN/SSIGN (localized) risk score. The relatively small number of patients in the high-risk groups were combined with the intermediate group for subsequent analysis. The results revealed that high CTR1 expression was associated with a poor prognosis in the low (OS, $\mathrm{P}<0.001$; DFS, $\mathrm{P}=0.016$; Fig. 2E and $\mathrm{F}$, respectively) 

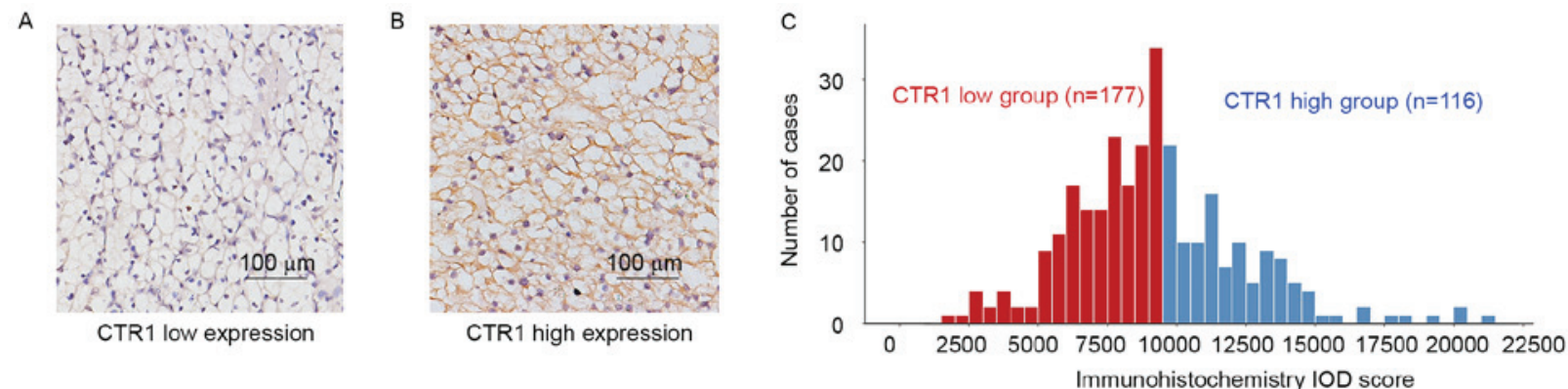

Figure 1. A CTR1 immunostaining in ccRCC. Representative images of (A) low CTR1 expression; (B) high CTR1 expression. Magnification, x200. (C) Frequency distribution of CTR1 immunohistochemistry integrated optical density scores in 293 ccRCC samples. ccRCC, clear cell renal cell carcinoma; CTR1, copper transporter 1; IOD, integrated optical density.

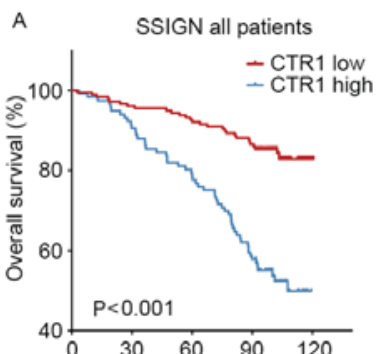

No. at risk Months after surgery

CTR1 low

$\begin{array}{rrrrr}177 & 170 & 163 & 153 & 2 \\ \text { CTR1 high } & & & & \end{array}$

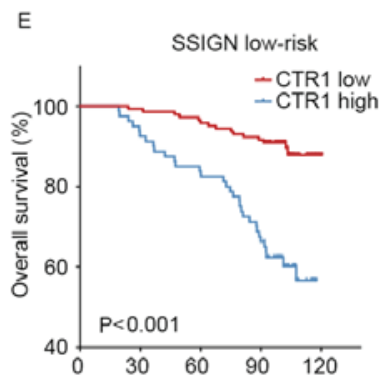

No. at risk Months after surgery

CTR1 low

$\begin{array}{rrrrr}144 & 143 & 138 & 132 & 2\end{array}$

$\begin{array}{lllll}80 & 74 & 67 & 53 & 0\end{array}$

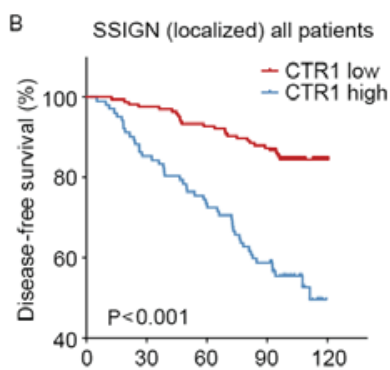

No. at risk Months after surgery CTR1 low $\begin{array}{rllll}166 & 162 & 154 & 145 & 2 \\ \text { CTR1 high } & & & & \end{array}$ $\begin{array}{lllll}102 & 87 & 75 & 60 & 0\end{array}$

F

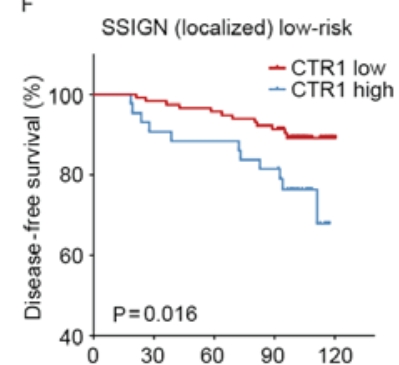

No. at risk Months after surgery

CTR1 low

$\begin{array}{rllll}116 & 114 & 111 & 106 & 2\end{array}$

$\begin{array}{lllll}43 & 39 & 38 & 35 & 0\end{array}$
C
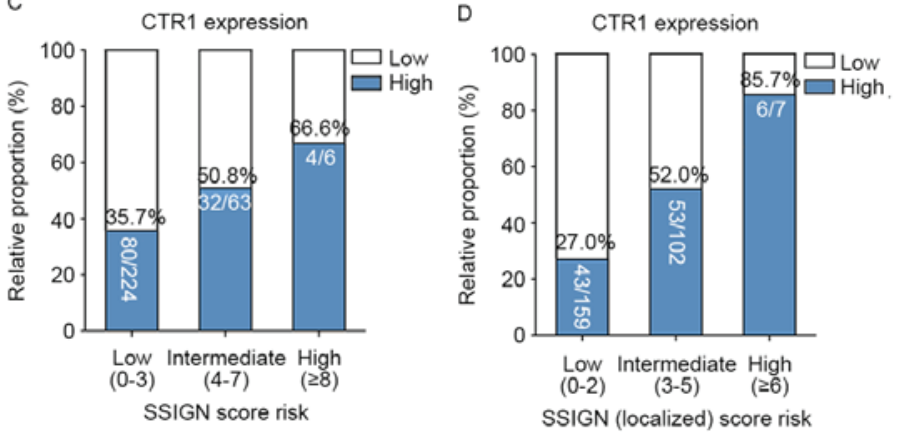

G

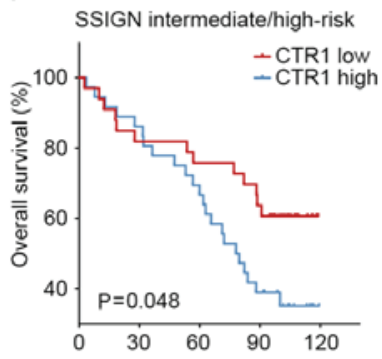

No. at risk Months after surgery

$\begin{array}{rllll}\text { CTR1 low } & & & & \\ \text { M } & 27 & 25 & 21 & 0\end{array}$

$\begin{array}{lllll}36 & 31 & 24 & 14 & 0\end{array}$

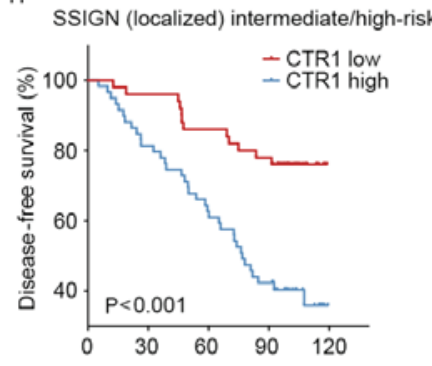

No. at risk Months after surgery

CTR1 low

$\begin{array}{rllll}50 & 48 & 43 & 39 & 0 \\ \text { CTR1 high } & & & & \end{array}$

$\begin{array}{lllll}48 & 37 & 25 & 0\end{array}$

Figure 2. CTR1 expression stratified by SSIGN/SSIGN (localized) score and Kaplan-Meier analyses of OS/DFS. (A) OS and (B) DFS rates of all patients according to CTR1 expression. Relative proportions of CTR1 expression status in three risk groups according to the (C) SSIGN and (D) SSIGN (localized) score. High CTR1 expression rates were elevated as the risks increased. (E) OS and (F) DFS rates of low-risk patients according to CTR1 expression. (G) OS and (H) DFS rates of intermediate/high-risk patients according to CTR1 expression. CTR1, copper transporter 1; SSIGN, Mayo Clinic stage, size, grade and necrosis; OS, overall survival; DFS, disease-free survival.

and intermediate/high (OS, P=0.048; DFS, P<0.001; Fig. 2G and $\mathrm{H}$, respectively) risk groups.

Univariate and multivariate analyses. A univariate analysis highlighted the prognostic value of high CTR1 expression in the prediction of a poor OS $(\mathrm{P}<0.001)$ and DFS $(\mathrm{P}<0.001)$ (Table II). Due to the small prognostic difference between Fuhrman grades 1 and 2, they were combined into one category. A multivariate analysis was performed, including the parameters with univariate statistical significance, in which tumor size and TNM stage were ruled out as potential confounding factors for $\mathrm{T}$ stage and distant metastasis. The age of patients was also removed, since it did not meet statistical significance subsequent to adjusting for other parameters. The analysis revealed that high expression of CTR1 in ccRCC was associated with a higher risk of mortality (hazard ratio, 2.291; 95\% CI, 1.389-3.777; P<0.001) and a shorter DFS time (hazard ratio, 2.210; 95\% CI, 1.299-3.759; $\mathrm{P}=0.003$; Table III). These results suggested that high tumor CTR1 expression was an independent poor prognostic marker in ccRCC adjusted with T stage, distant metastasis, Fuhrman grade, necrosis and ECOG PS.

Extension of established prognostic models with CTR1 expression. CTR1 expression was added as a binary variable to 
Table III. Multivariate analyses of characteristics associated with OS and DFS.

\begin{tabular}{|c|c|c|c|c|c|c|}
\hline \multirow[b]{2}{*}{ Variables } & \multicolumn{3}{|c|}{ OS (n=293) } & \multicolumn{3}{|c|}{ DFS (n=268) } \\
\hline & HR & $95 \% \mathrm{CI}$ & P-value ${ }^{a}$ & HR & $95 \% \mathrm{CI}$ & P-value ${ }^{a}$ \\
\hline T stage & & & 0.008 & & & $<0.001$ \\
\hline $\mathrm{T} 1$ & RV & - & - & RV & - & - \\
\hline $\mathrm{T} 2$ & 2.356 & $1.380-4.021$ & 0.002 & 2.516 & $1.155-5.483$ & 0.020 \\
\hline $\mathrm{T} 3$ & 2.406 & $1.200-4.824$ & 0.013 & 2.735 & $1.542-4.853$ & 0.001 \\
\hline $\mathrm{T} 4$ & 3.319 & $0.885-12.447$ & 0.075 & 8.353 & $2.457-28.393$ & 0.001 \\
\hline \multicolumn{7}{|l|}{ Distant metastasis } \\
\hline Yes vs. no & 3.534 & $1.841-6.784$ & $<0.001$ & - & - & - \\
\hline Fuhrman grade & & & 0.002 & & & $<0.001$ \\
\hline $1-2$ & RV & - & - & RV & - & - \\
\hline 3 & 2.230 & $1.298-3.832$ & 0.004 & 2.839 & $1.553-5.190$ & 0.001 \\
\hline 4 & 4.125 & $1.182-14.401$ & 0.026 & 4.810 & $1.342-17.242$ & 0.016 \\
\hline \multicolumn{7}{|l|}{ Necrosis } \\
\hline Present vs. absent & 2.010 & $1.151-3.511$ & 0.014 & 2.087 & $1.177-3.701$ & 0.012 \\
\hline ECOG PS & & & 0.005 & & & 0.012 \\
\hline 0 & RV & - & - & RV & - & - \\
\hline 1 & 2.130 & $1.301-3.487$ & 0.003 & 1.957 & $1.115-3.434$ & 0.019 \\
\hline 2 & 2.310 & $0.789-6.760$ & 0.126 & 3.233 & $0.980-10.667$ & 0.054 \\
\hline 3 & 3.514 & $1.169-10.568$ & 0.025 & 3.236 & $1.048-9.993$ & 0.041 \\
\hline \multicolumn{7}{|c|}{ Tumor CTR 1 expression } \\
\hline Low vs. high & 2.291 & $1.389-3.777$ & 0.001 & 2.210 & $1.299-3.759$ & 0.003 \\
\hline
\end{tabular}

${ }^{a}$ Data obtained from the Cox proportional hazards model. $\mathrm{P}<0.05$ was regarded as statistically significant. OS, overall survival; DFS, disease-free survival; HR, hazard ratio; CI, confidence interval; RV, reference value; CTR1, copper transporter 1.

Table IV. Comparison of the predictive accuracy of the prognostic models.

\begin{tabular}{|c|c|c|c|c|c|c|}
\hline \multirow[b]{2}{*}{ Models } & \multicolumn{3}{|c|}{ Overall survival $(n=293)$} & \multicolumn{3}{|c|}{ Disease free survival $(\mathrm{n}=268)$} \\
\hline & C-index & P-value ${ }^{a}$ & AIC & C-index & P-value & AIC \\
\hline CTR1 & 0.654 & & 878.03 & 0.664 & & 736.39 \\
\hline TNM & 0.702 & & 865.51 & 0.653 & & 746.76 \\
\hline TNM + CTR1 & 0.750 & 0.003 & 849.72 & 0.722 & 0.015 & 726.30 \\
\hline SSIGN & 0.740 & & 858.94 & 0.725 & & 722.78 \\
\hline SSIGN + CTR1 & 0.779 & 0.024 & 839.81 & 0.763 & 0.090 & 705.21 \\
\hline UISS & 0.722 & & 861.26 & 0.713 & & 726.29 \\
\hline UISS + CTR 1 & 0.766 & 0.021 & 842.16 & 0.750 & 0.021 & 711.79 \\
\hline
\end{tabular}

${ }^{\mathrm{a}} \mathrm{C}$-index compared with the original model without CTR1 expression. C-index, concordance index; AIC, Akaike information criterion; SSIGN, Mayo clinic stage, size, grade and necrosis score; UISS, University of California Integrated Staging System; TNM, tumor-node-metastasis; CTR1, copper transporter 1 .

well-established prognostic systems (TNM stage, SSIGN and UISS) and it was investigated whether this biomarker could improve their prognostic power. As included in Table IV, subsequent to being integrated with CTR1 expression, the c-indexes of all existing models increased markedly for OS $(\mathrm{P}=0.003$ for TNM, $\mathrm{P}=0.024$ for SSIGN, $\mathrm{P}=0.021$ for UISS, respectively) and DFS analyses $(\mathrm{P}=0.015$ for $\mathrm{T}$ stage, $\mathrm{P}=0.090$ for $\mathrm{SSIGN}$,
$\mathrm{P}=0.021$ for UISS, respectively). The AIC was also calculated for each model; models that included CTR1 status presented a lower score than the models that did not include CTR1 status, indicating the potential prognostic value of CTR1.

Nomograms for predicting OS and DFS in patients with $c c R C C$. Based on the results arising from multivariate 
A

Points

T stage

Distant metastasis

Fuhrman nuclear grade

Necrosis

ECOG PS

CTR1 expression

Total points

5-year OS probability

8-year OS probability

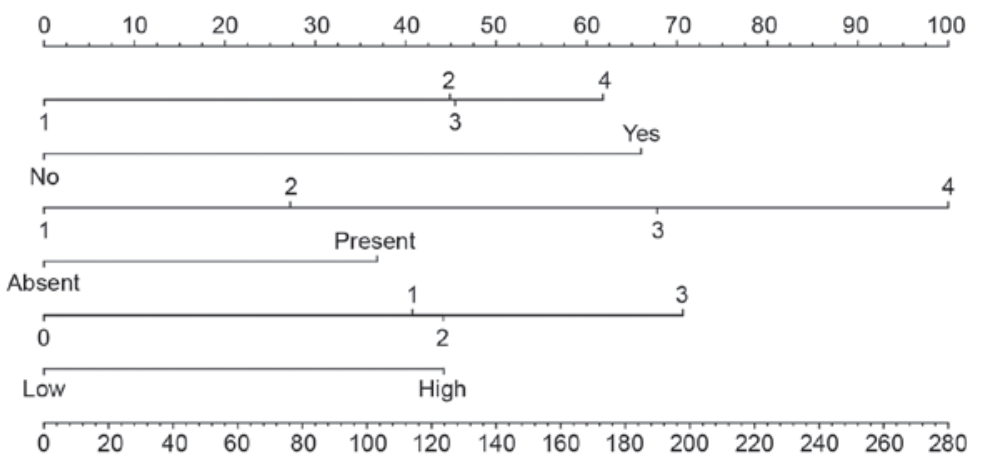

$\begin{array}{lllllllll}0.9 & 0.8 & 0.7 & 0.6 & 0.5 & 0.4 & 0.3 & 0.2 & 0.1\end{array}$
B

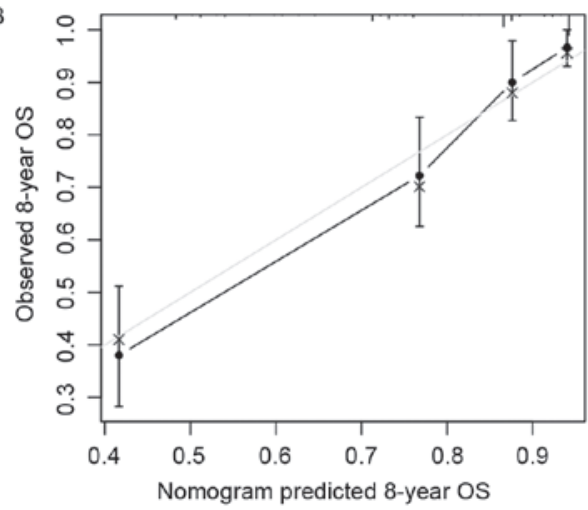

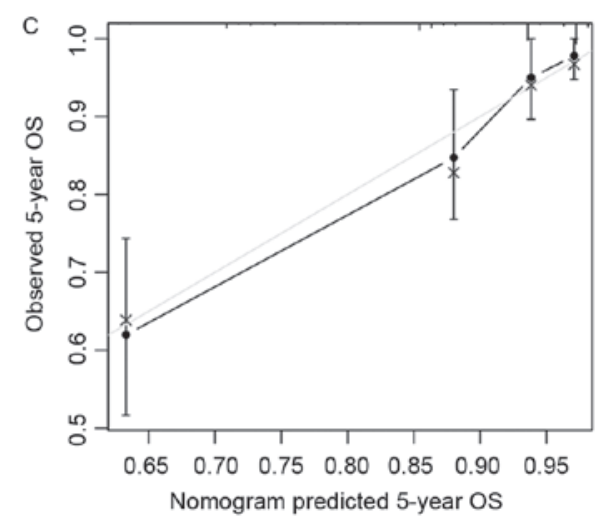

Figure 3. Nomogram for predicting 8- and 5-year overall survival in patients with clear cell renal cell carcinoma. (A) Nomogram for predicting clinical outcomes, integrating T stage, distant metastasis, Fuhrman nuclear grade, necrosis, ECOG PS and tumor CTR1 expression. (B) Calibration plot for predicted and observed 8-year OS rate. (C) Calibration plot for predicted and observed 5-year OS rate. Grey line, ideal model; vertical bars, $95 \%$ confidence interval. ECOG PS, Eastern Cooperative Oncology Group performance status; CRT1, copper transporter 1; OS, overall survival.

analysis, two nomograms were constructed for predicting the 5- and 8-year OS and DFS rates of patients with ccRCC (Figs. 3A and 4A). The predictors included were $\mathrm{T}$ stage, distant metastasis, Fuhrman grade, necrosis status, ECOG PS and tumor CTR1 expression. Bootstrap validations were performed for calibration (Figs. 3B and C; 4B and C). The Harrell's c-indexes were 0.805 (95\% CI, 0.764-0.846) and 0.787 (95\% CI, 0.736-0.838) for OS and DFS prediction, respectively.

\section{Discussion}

ccRCC is well elucidated for its VHL/HIF dysregulation and downstream signal abnormalities (19). Furthermore, CTR1, as a high-affinity copper transporter mediating cellular copper upregulation, is considered to promote tumor angiogenesis and progression indirectly through the HIF pathway (15). Therefore, the prognostic role of CTR1 expression was explored in patients with ccRCC.

Through IHC, CTR1 was identified on the cell plasma membrane and intracellular area in ccRCC specimens, consistent with previous findings (28). In addition, high tumor CTR1 expression was positively associated with various clinical characteristics and a higher SSIGN score, and could be used to stratify the outcome of patients in different SSIGN risk groups. CTR1 also exhibited an independent negative prognostic effect on the predictions of OS and DFS of patients with ccRCC, adjusted with other parameters. Adding CTR1 expression information into existing prognostic models, including TNM, SSIGN and UISS, noticeably enhanced their prognostic power. Finally, two nomograms were generated by integrating CTR1 expression with other clinical parameters to predict the OS and DFS of patients with ccRCC. The c-indexes were 0.805 and 0.787 for OS and DFS, respectively, revealing an improved prognostic ability, compared with existing survival models, for the present cohort.

CTR1 is a transmembrane glycoprotein encoded by gene hCTR1 (SLC31A1), located on chromosome 9q31/32 together with its homolog hCTR2 (SLC31A2) (9). Although previous study has predominantly emphasized the platinum uptake capability of CTR1, which may amplify the therapeutic effects of platinum-based chemotherapies in ovarian and lung cancer (29), its primary copper regulation ability and downstream signaling still serve important roles in tumor progression.

CTR1 functions as a stimulator for tumor angiogenesis based on its copper intake ability and the activation of the HIF-1 $\alpha$ /VEGF pathway by copper (16). Furthermore, evidence 


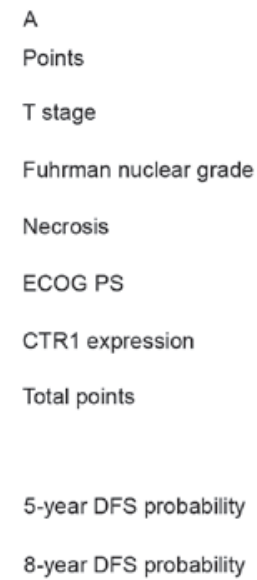

8-year DFS probability

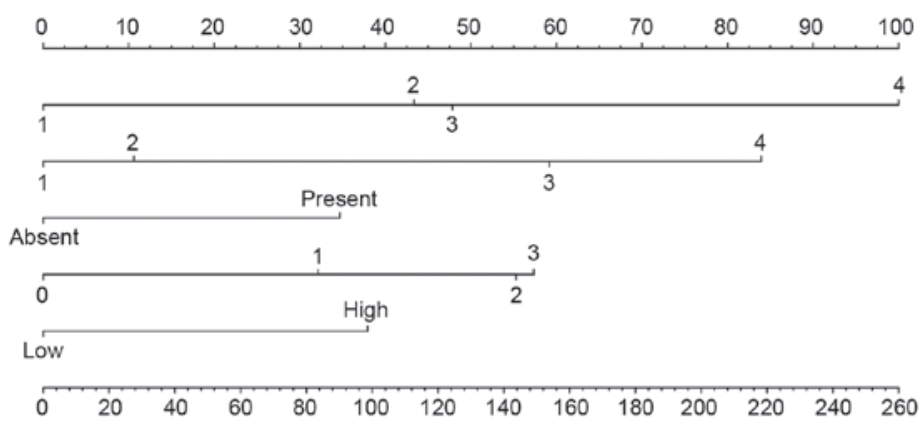

\begin{tabular}{|c|c|c|c|c|c|c|c|}
\hline & 0.9 & & 0.8 & 0.7 & 0.6 & $\begin{array}{llll}0.5 & 0.4 & 0.3 & 0.2\end{array}$ & 20.1 \\
\hline 0.9 & 0.8 & 0.7 & 0.6 & 0.50 .4 & 40.3 & $0.2 \quad 0.1$ & \\
\hline
\end{tabular}

B

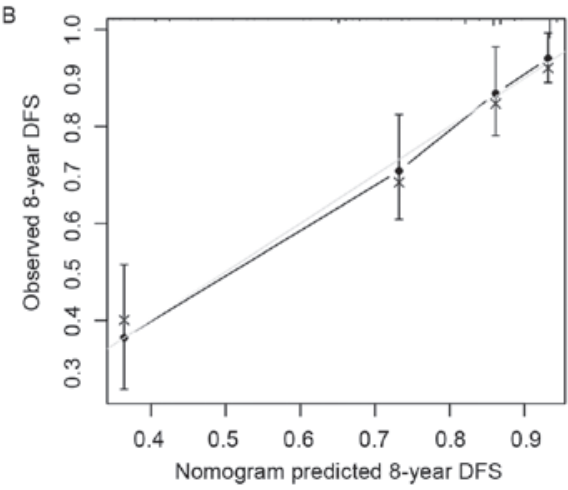

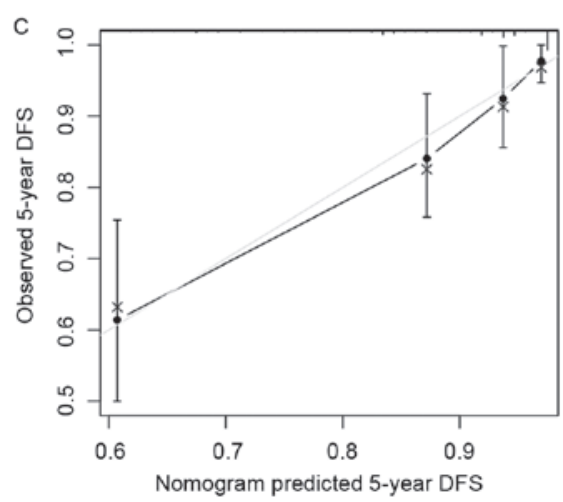

Figure 4. Nomogram for predicting 8- and 5-year DFS in patients with clear cell renal cell carcinoma. (A) Nomogram for predicting clinical outcomes integrating T stage, Fuhrman nuclear grade, necrosis, ECOG PS and tumor CTR1 expression. (B) Calibration plot for predicted and observed 8-year DFS rate. (C) Calibration plot for predicted and observed 5-year DFS rate. Grey line, ideal model; vertical bars, 95\% confidence interval. DFS, disease-free survival; ECOG PS, Eastern Cooperative Oncology Group performance status; CTR1, copper transporter 1.

indicates that CTR1 incorporates with HIF-2 $\alpha$ (30), which may be a more important regulator for ccRCC proliferation (31). In addition, knockdown of CTR1 in human breast cancer cells was identified to inhibit EMT formation through HIF1- $\alpha$ inhibition (15). The mitogen-activated protein kinase (MAPK) kinase 1/extracellular signal-regulated kinase (ERK) pathway can also be regulated by CTR1 through enhancement of ERK1/2 phosphorylation by copper (32), and the aberrant activation of MAPKs was regarded as an important mechanism for RCC mechanistic target of rapamycin (mTOR) inhibitor resistance (33).

Since dysregulation of the VHL/HIF pathway is a dominant driving force for ccRCC initiation, and evidence suggests that copper and ceruloplasmin levels are upregulated in ccRCC (34), it is tempting to speculate that in the present study, the poor prognostic effect of high CTR1 expression may be associated with the copper level and the HIF pathway in ccRCC cells. However, this hypothesis remains to be validated rigorously through further experiments.

Our previous study revealed that the expression of another copper transporter, CTR2, was decreased in ccRCC compared with peritumoral tissue, and low tumor CTR2 expression predicted a reduced OS and DFS time for patients with ccRCC (35). CTR2 is a low affinity copper transporter and a previous study has identified its opposite role against CTR1, based on evidence that CTR2 may aid the degradation of CTR1 into a cleaved form, which imports copper less efficiently (36). The opposite prognostic roles of CTR1 and CTR2 in platinum-based chemotherapy resistance also support this theory (37). However, a previous study has also suggested that CTR1 is essential to maintain the stability of CTR2 (38). The present study revealed that high CTR1 expression indicated a poor prognosis for patients with ccRCC, which is in contrary to CTR2. The underlying mechanisms require further study, and whether CTR1 expression can provide additional prognosis value when considered with CTR2 expression is under investigation.

For metastatic RCC, antiangiogenic therapies, including tyrosine kinase and mTOR inhibitors targeting the HIF pathway, may prolong the patient survival time. Since copper is also associated with the promotion of tumor angiogenesis and progression, a depletion of copper may have therapeutic effects. A phase II clinical trial was performed with tetrathiomolybdate, an oral copper chelator, for treating patients with metastatic RCC (39). However, the results were limited to stable disease for a median of 34 weeks in one-third of the patients. As CTR1 exhibited a significant prognostic effect in patients with ccRCC in the present study, future studies should investigate whether this molecule can identify the sensitivity of patients with ccRCC to targeted therapies. In addition, treatments targeting CTR1 and associated molecules may provide a novel perspective in ccRCC management. 
The major limitations of the present study are the retrospective nature of the study and the relatively small sample size. A multicenter prospective study is required to validate the results in a larger population in the future. SSIGN was applied rather than UISS in the subgroup OS analysis in Fig. 2, as the former was more sensitive in risk stratification and prognosis prediction in the database (c-index 0.740 vs. 0.722; Table IV), although it was initially designed for predicting cancer-specific survival, defined as the time from nephrectomy to disease-induced mortality. In addition, the proportion of patients with advanced ccRCC was markedly smaller in the present cohort compared with other clinical databases and there were only 2 patients with positive lymph node metastasis, which may have influenced the non-significance of $\mathrm{N}$ stage in the univariate analysis. In addition, several HIF-associated molecules, including HIF-1 $\alpha$, HIF-2 $\alpha$, VEGF and EMT-associated proteins have not been analyzed and incorporated with CTR1 in the present study. A number of other survival predictors, including the positive margin rates, sarcomatoid differentiation, and the diabetes status and body mass index of patients have not been involved in the multivariate analysis and merit further study.

In conclusion, the present study indicated that the high expression of tumor CTR 1 is associated with poor survival in patients with ccRCC. This novel biomarker may be incorporated with other clinical parameters to form nomograms for the improved prediction of prognosis.

\section{Acknowledgements}

The authors thank Dr Yuan Ji, Dr Jun Hou and Ms Haiying Zeng (Department of Pathology, Zhongshan Hospital of Fudan University, Fudan, China) for diagnosis confirmation and technical assistance, respectively. The present study was funded by grants from the National Basic Research Program of China (grant no. 2012CB822104), the National Key Projects for Infectious Diseases of China (grant nos. 2012ZX10002012-007 and 2016ZX10002018-008), the National Natural Science Foundation of China (grant nos. 31100629, 31270863, 81372755, 31470794, 81401988, 81402082, 81402085, 81471621, 81472227, $81472376,31570803,81501999$ and 81572352) and the Program for New Century Excellent Talents in University (grant no. NCET-13-0146). All study sponsors had no role in the study design or the collection, analysis and interpretation of data.

\section{References}

1. Siegel R, Ma J, Zou Z and Jemal A: Cancer statistics, 2014. CA Cancer J Clin 64: 9-29, 2014.

2. Lam JS, Shvarts O, Leppert JT, Figlin RA and Belldegrun AS: Renal cell carcinoma 2005: New frontiers in staging, prognostication and targeted molecular therapy. J Urol 173: 1853-1862, 2005.

3. Edge SB and Compton CC: The American Joint Committee on Cancer: The 7th edition of the AJCC cancer staging manual and the future of TNM. Ann Surg Oncol 17: 1471-1474, 2010.

4. Fuhrman SA, Lasky LC and Limas C: Prognostic significance of morphologic parameters in renal cell carcinoma. Am J Surg Pathol 6: 655-663, 1982.

5. Frank I, Blute ML, Cheville JC, Lohse CM, Weaver AL and Zincke H: An outcome prediction model for patients with clear cell renal cell carcinoma treated with radical nephrectomy based on tumor stage, size, grade and necrosis: The SSIGN score. J Urol 168: 2395-2400, 2002.
6. Sun M, Shariat SF, Cheng C, Ficarra V, Murai M, Oudard S, Pantuck AJ, Zigeuner R and Karakiewicz PI: Prognostic factors and predictive models in renal cell carcinoma: A contemporary review. Eur Urol 60: 644-661, 2011.

7. Volpe A and Patard JJ: Prognostic factors in renal cell carcinoma. World J Urol 28: 319-327, 2010.

8. Shariat SF and Xylinas E: Biomarkers in personalised treatment of renal-cell carcinoma. Lancet Oncol 13: 751-752, 2012.

9. Zhou B and Gitschier J: hCTR1: A human gene for copper uptake identified by complementation in yeast. Proc Natl Acad Sci USA 94: 7481-7486, 1997.

10. Lee J, Prohaska JR, Dagenais SL, Glover TW and Thiele DJ: Isolation of a murine copper transporter gene, tissue specific expression and functional complementation of a yeast copper transport mutant. Gene 254: 87-96, 2000.

11. Hu GF: Copper stimulates proliferation of human endothelial cells under culture. J Cell Biochem 69: 326-335, 1998.

12. Díez M, Arroyo M, Cerdàn FJ, Muñoz M, Martin MA and Balibrea JL: Serum and tissue trace metal levels in lung cancer. Oncology 46: 230-234, 1989.

13. Kuo HW, Chen SF, Wu CC, Chen DR and Lee JH: Serum and tissue trace elements in patients with breast cancer in Taiwan. Biol Trace Elem Res 89: 1-11, 2002.

14. Martin F, Linden T, Katschinski DM, Oehme F, Flamme I, Mukhopadhyay CK, Eckhardt K, Tröger J, Barth S, Camenisch G and Wenger RH: Copper-dependent activation of hypoxia-inducible factor (HIF)-1: Implications for ceruloplasmin regulation. Blood 105: 4613-4619, 2005.

15. Li S, Zhang J, Yang H, Wu C, Dang X and Liu Y: Copper depletion inhibits CoCl2-induced aggressive phenotype of MCF-7 cells via downregulation of HIF-1 and inhibition of Snail/Twist-mediated epithelial-mesenchymal transition. Sci Rep 5: 12410, 2015.

16. Narayanan G, R BS, Vuyyuru H, Muthuvel B and Konerirajapuram Natrajan S: CTR1 silencing inhibits angiogenesis by limiting copper entry into endothelial cells. PLoS One 8: e71982, 2013.

17. Brewer GJ, Dick RD, Grover DK, LeClaire V, Tseng M, Wicha M, Pienta K, Redman BG, Jahan T, Sondak VK, et al: Treatment of metastatic cancer with tetrathiomolybdate, an anticopper, antiangiogenic agent: Phase I study. Clin Cancer Res 6: 1-10, 2000.

18. Escudier B, Porta C, Schmidinger M, Rioux-Leclercq N, Bex A, Khoo V, Gruenvald V and Horwich A; ESMO Guidelines Committee: Renal cell carcinoma: ESMO Clinical Practice Guidelines for diagnosis, treatment and follow-up. Ann Oncol 27 (Suppl 5): v58-v68, 2016.

19. Cohen HT and McGovern FJ: Renal-cell carcinoma. N Engl J Med 353: 2477-2490, 2005.

20. Buccheri G, Ferrigno D and Tamburini M: Karnofsky and ECOG performance status scoring in lung cancer: A prospective, longitudinal study of 536 patients from a single institution. Eur J Cancer 32: 1135-1141, 1996.

21. Lopez-Beltran A, Bassi P, Pavone-Macaluso M and Montironi R: Handling and pathology reporting of specimens with carcinoma of the urinary bladder, ureter, and renal pelvis. Eur Urol 45: 257-266, 2004.

22. Chang Y, An H, Xu L, Zhu Y, Yang Y, Lin Z and Xu J: Systemic inflammation score predicts postoperative prognosis of patients with clear-cell renal cell carcinoma. Br J Cancer 113: 626-633, 2015.

23. Leibovich BC, Blute ML, Cheville JC, Lohse CM, Frank I, Kwon ED, Weaver AL, Parker AS and Zincke H: Prediction of progression after radical nephrectomy for patients with clear cell renal cell carcinoma: A stratification tool for prospective clinical trials. Cancer 97: 1663-1671, 2003.

24. Pan D, Xu L, Liu H, Zhang W, Zhu Y, Xu J and Gu J: Interleukin-11 receptor predicts post-operative clinical outcome in patients with early-stage clear-cell renal cell carcinoma. Jpn J Clin Oncol 45: 202-209, 2015.

25. Wu K, Xu L, Zhang L, Lin Z and Hou J: High Jagged1 expression predicts poor outcome in clear cell renal cell carcinoma. Jpn J Clin Oncol 41: 411-416, 2011.

26. Camp RL, Dolled-Filhart M and Rimm DL: X-tile: A new bio-informatics tool for biomarker assessment and outcome-based cut-point optimization. Clin Cancer Res 10: 7252-7259, 2004.

27. Harrell FE Jr, Califf RM, Pryor DB, Lee KL and Rosati RA: Evaluating the yield of medical tests. JAMA 247: 2543-2546, 1982.

28. Klomp AE, Tops BB, Van Denberg IE, Berger R and Klomp LW: Biochemical characterization and subcellular localization of human copper transporter 1 (hCTR1). Biochem J 364: 497-505, 2002 . 
29. Howell SB, Safaei R, Larson CA and Sailor MJ: Copper transporters and the cellular pharmacology of the platinum-containing cancer drugs. Mol Pharmacol 77: 887-894, 2010.

30. Pourvali K, Matak P, Latunde-Dada GO, Solomou S Mastrogiannaki M, Peyssonnaux C and Sharp PA: Basa expression of copper transporter 1 in intestinal epithelial cells is regulated by hypoxia-inducible factor $2 \alpha$. FEBS Lett 586 : 2423-2427, 2012.

31. Gordan JD, Bertout JA, Hu CJ, Diehl JA and Simon MC: HIF-2alpha promotes hypoxic cell proliferation by enhancing c-myc transcriptional activity. Cancer Cell 11: 335-347, 2007.

32. Brady DC, Crowe MS, Turski ML, Hobbs GA, Yao X Chaikuad A, Knapp S, Xiao K, Campbell SL, Thiele DJ and Counter CM: Copper is required for oncogenic BRAF signalling and tumorigenesis. Nature 509: 492-496, 2014.

33. Bailey ST, Zhou B, Damrauer JS, Krishnan B, Wilson HL, Smith AM, Li M, Yeh JJ and Kim WY: mTOR inhibition induces compensatory, therapeutically targetable MEK activation in renal cell carcinoma. PLoS One 9: e104413, 2014.

34. Stassar MJ, Devitt G, Brosius M, Rinnab L, Prang J, Schradin T, Simon J, Petersen S, Kopp-Schneider A and Zöller M: Identification of human renal cell carcinoma associated genes by suppression subtractive hybridization. Br J Cancer 85: 1372-1382, 2001.
35. Xia Y, Liu L, Long Q, Bai Q, Wang J, Xu J and Guo J: Decreased expression of CTR2 predicts poor prognosis of patients with clear cell renal cell carcinoma. Urol Oncol 34: 5.el-e9, 2016.

36. Ohrvik H, Nose Y, Wood LK, Kim BE, Gleber SC, Ralle M and Thiele DJ: Ctr2 regulates biogenesis of a cleaved form of mammalian Ctrl metal transporter lacking the copper- and cisplatin-binding ecto-domain. Proc Natl Acad Sci USA 110: E4279-E4288, 2013.

37. Lee YY, Choi CH, Do IG, Song SY, Lee W, Park HS, Song TJ, Kim MK, Kim TJ, Lee JW, et al: Prognostic value of the copper transporters, CTR1 and CTR2, in patients with ovarian carcinoma receiving platinum-based chemotherapy. Gynecol Oncol 122: 361-365, 2011

38. Tsai CY, Liebig JK, Tsigelny IF and Howell SB: The copper transporter 1 (CTR1) is required to maintain the stability of copper transporter 2 (CTR2). Metallomics 7: 1477-1487, 2015.

39. Redman BG, Esper P, Pan Q, Dunn RL, Hussain HK, Chenevert T, Brewer GJ and Merajver SD: Phase II trial of tetrathiomolybdate in patients with advanced kidney cancer. Clin Cancer Res 9: 1666-1672, 2003 